\title{
Diffusion-weighted MRS of acetate in the rat brain
}

\author{
Masoumeh Dehghani ${ }^{1}$ | Nicolas Kunz ${ }^{2}$ | Bernard Lanz ${ }^{1,3}$ | Hikari A.I. Yoshihara ${ }^{4}$ (D) | \\ Rolf Gruetter ${ }^{1,5,6}$
}

${ }^{1}$ Laboratoire d'imagerie fonctionnelle et métabolique (LIFMET), École polytechnique fédérale de Lausanne (EPFL), Lausanne, Switzerland

${ }^{2}$ Centre d'Imagerie BioMédicale (CIBM)-AIT, École polytechnique fédérale de Lausanne (EPFL), Lausanne, Switzerland

${ }^{3}$ Sir Peter Mansfield Imaging Centre, School of Physics and Astronomy, University of Nottingham, Nottingham, UK

${ }^{4}$ Service de Cardiologie, Centre Hospitalier Universitaire Vaudois (CHUV), Lausanne, Switzerland

${ }^{5}$ Centre d'Imagerie BioMédicale (CIBM), École polytechnique fédérale de Lausanne (EPFL), Lausanne, Switzerland

${ }^{6}$ Department of Radiology, Université de Lausanne (UNIL), Lausanne, Switzerland

\section{Correspondence}

Masoumeh Dehghani, Laboratoire d'imagerie fonctionnelle et métabolique (LIFMET), École polytechnique fédérale de Lausanne (EPFL), 1015-Lausanne, Switzerland.

Email: masoumeh.dehghani@epfl.ch; dm.dehghani@gmail.com

Funding information

Swiss National Science Foundation, Grant/ Award Number: 149983; Centre d'Imagerie BioMédicale (CIBM)

\section{1 | INTRODUCTION}

Acetate has been proposed as an astrocyte-specific energy substrate for the brain and is an attractive alternative to glucose for in vitro and in vivo nuclear magnetic resonance (NMR) studies of brain metabolism. ${ }^{1-6}$ The uptake of infused labeled acetate by only a single cell type in the brain (glial compartment) can simplify the modeling and increase the sensitivity of the measurement of the glutamate-glutamine cycle in the brain relative to the use of glucose, which is taken up by both neuronal and glial compartments alike.

To take full advantage of the dynamic information available from in vivo ${ }^{13} \mathrm{C}$ NMR spectroscopy and to perform dynamic metabolic modeling of the tricarboxylic acid (TCA) cycle activity and glutamate/glutamine cycling, prior knowledge of the transport and uptake kinetics of infused acetate across the blood-brain barrier is required. Quantitative measurement of acetate transport in the brain has often been performed by fitting the measured NMR data to the Michaelis-Menten model which requires the knowledge of the distribution volume $\left(V_{d}\right)$ of acetate in the brain. ${ }^{7,8}$

Abbreviations used: Ace, acetate; ADC, apparent diffusion coefficient; $b$-value, diffusion weighting strength; $\mathrm{CHESS}$ pulse, chemical shift-selective pulse; $\mathrm{Cr}$, creatine; CRLB, Cramér-Rao lower bound; $D_{\text {fast }}^{\text {App }} / D_{\text {slow }}^{\text {App }}$, apparent diffusion coefficients of the fast/slow decaying signal components; $D_{\text {mono }}^{\text {App }}$, apparent mono-exponential diffusion coefficient; $D_{\text {free }}$, self-diffusion coefficient; DW-MRS, diffusion-weighted magnetic resonance spectroscopy; FID, free induction decay; GABA, $\gamma$-aminobutyric acid; IR, inversion recovery; $\mathrm{MCT}$, monocarboxylate transporter; $\mathrm{MM}$, macromolecules; NAA, $N$-acetylaspartate; NMR, nuclear magnetic resonance; $p_{\text {slow }}^{\text {App }}$ relative contribution of the slow component in the metabolite signal; PCr, phosphocreatine; $p_{0}^{\text {extra }}$, extracellular fraction; $r_{\text {fast }}^{\text {Applow }}$, apparent mean displacements of fast/slow component; RF, radiofrequency; SNR, signal-to-noise ratio; STEAM, stimulated echo acquisition mode; TCA cycle, tricarboxylic acid cycle; TE, echo time; TI, inversion time; TM, mixing time; TR, repetition time; $V_{d}$, distribution volume; VOI, volume of interest 
However, the distribution volume of acetate in the rat brain during prolonged acetate infusion is not known. To study the transport and utilization of acetate in the rat brain, Deelchand et al. ${ }^{8}$ assumed the same distribution volume for acetate as for glucose $\left(V_{d}=0.77 \mathrm{~mL} / \mathrm{g}\right)$, whereas Patel et al. ${ }^{7}$ considered a smaller distribution volume for acetate, equal to $0.32 \mathrm{~mL} / \mathrm{g}$, based on the hypothesis that acetate is mainly localized in glial cells (whereas glucose is found in glial and neuronal cells). The assumed value for $V_{d}$ impacts on the reported parameters for acetate transport kinetics. To our knowledge, the acetate distribution and relative partitioning into the intracellular and extracellular compartments of the brain, measured using the non-invasive NMR modality, has not been reported.

Most in vivo NMR spectroscopy modalities do not distinguish between intracellular and extracellular contributions and provide information about the whole-tissue properties, such as metabolite concentrations in intact tissue., ${ }^{1,9-12}$ However, this information is important for accurate metabolic modeling. By measuring the translational displacement of metabolites in tissue and providing information about the cellular environment, diffusion-weighted magnetic resonance spectroscopy (DW-MRS) could help in this regard (for reviews, see Cao \& Wu, ${ }^{13} \mathrm{Nicolay}$ et al. ${ }^{14}$ and Ronen \& Valette, ${ }^{15}$ and references therein). DW-MRS can differentiate between the signal originating from intracellular and extracellular compartments based on their different diffusion properties. ${ }^{16}$ Therefore, DW-MRS is likely to yield insights into the distribution volume of acetate in the rat brain.

${ }^{1} \mathrm{H}$ DW-MRS remains a very challenging technique from the perspective of the acquisition of robust and reproducible DW-MRS data and their subsequent processing and quantification. DW-MRS contrast is based on the random translational displacement of a metabolite; therefore, any type of bulk motion could result in amplitude or phase fluctuation of the NMR signal. The influence of eddy currents on the spectral line shape is not particular to DW-MRS, but the application of strong diffusion-encoding gradient pulses significantly increases their effect. In diffusion measurements, it is important to note that all the gradient operations in the pulse sequence should be taken into account when estimating the diffusion weighting strength ( $b$-value), including the slice selection and crusher gradients as well as the main diffusion-encoding gradients.

A few studies have used DW-MRS to assess the diffusion properties of metabolites in the rat brain in vivo. ${ }^{17-22}$ Most studies have focused on the diffusion properties of particularly abundant metabolites, such as $\mathrm{N}$-acetylaspartate (NAA), which are predominantly located in the intracellular compartment. Pfeuffer et al. ${ }^{23}$ performed a DW-MRS study to test the hypothesis predicting a uniform distribution of infused glucose in the extracellular and intracellular spaces of the rat brain, and characterized the diffusion properties of 14 metabolites. The application of a similar strategy, ${ }^{1} \mathrm{H}$ DW-MRS of brain acetate under prolonged acetate infusion, could also provide information on the intrinsic diffusion properties of acetate in the brain and yield an insight into the cellular compartments that constrain its diffusional displacement.

The overlapping of metabolite resonances in in vivo spectra complicates the quantification of each individual metabolite. The challenge in the study of acetate diffusion behavior is the overlap of the acetate ${ }^{1} \mathrm{H}$-MRS resonance $(1.90 \mathrm{ppm})$ with a $\gamma$-aminobutyric acid (GABA) resonance at $1.89 \mathrm{ppm}$, which hinders an accurate estimation of the concentration and diffusivity of acetate. Therefore, the purpose of this study was to address the diffusion characteristics of acetate in the rat brain in vivo using DW-MRS techniques at steady-state concentration, whilst minimizing the contribution of the overlapping GABA resonance.

\section{2 | METHODS}

\section{1 | Animal preparation}

All experimental procedures involving animals were approved by the local veterinary authorities. Seven male Sprague-Dawley rats (200-235 g delivered from Charles River Laboratories, L'Arbresle Cedex, France) were prepared without fasting. Animals were intubated and ventilated with $2 \%$ isoflurane during surgery. Both femoral veins were catheterized for the administration of acetate and a-chloralose. Two arteries were cannulated for the continuous monitoring of physiology (blood pressure and heart rate) and periodic blood sampling for blood gas, plasma lactate and glucose concentration measurements. Following surgery, anesthesia was switched to intravenous a-chloralose administration (bolus of $80 \mathrm{mg} / \mathrm{kg}$ and continuous infusion rate of $26.7 \mathrm{mg} / \mathrm{kg} / \mathrm{h}$ ). Animals were placed in a home-built holder and the head position was fixed using ear rods and a bite-bar. The body temperature was maintained between 37.0 and $38^{\circ} \mathrm{C}$ with a temperature-regulated circulating water bath.

\subsection{Acetate infusion protocol}

The sodium acetate infusion protocol was optimized on the bench and in the magnet to reach a steady-state concentration of acetate in the rat brain in less than $30 \mathrm{~min}$, whilst keeping the animal within physiological limits throughout the study. After the adjustment of MRS parameters and shimming, a bolus of $1 \mathrm{~g} / \mathrm{kg}$ of sodium acetate $(1.6 \mathrm{M})$ was administered with an infusion rate decaying in a two-step process over 10 min (Figure 1). This bolus was followed by a continuous infusion of acetate at a rate of $1.4 \mathrm{~g} / \mathrm{kg} / \mathrm{h}$.

In order to avoid metabolic alkalosis in the blood of animals during the study, ${ }^{24,25}$ the acetate solution was infused at an acidic $\mathrm{pH}(\mathrm{pH} 5.0$ ) as used previously in rats. ${ }^{8,26}$ 


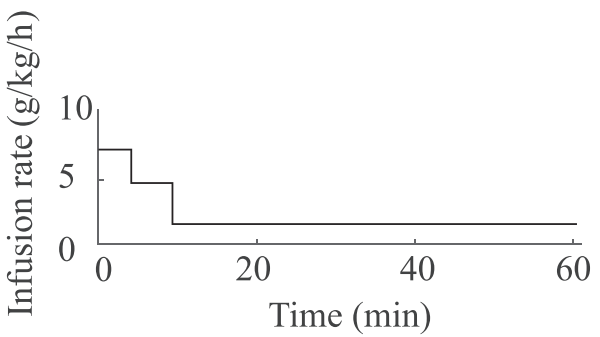

(A)

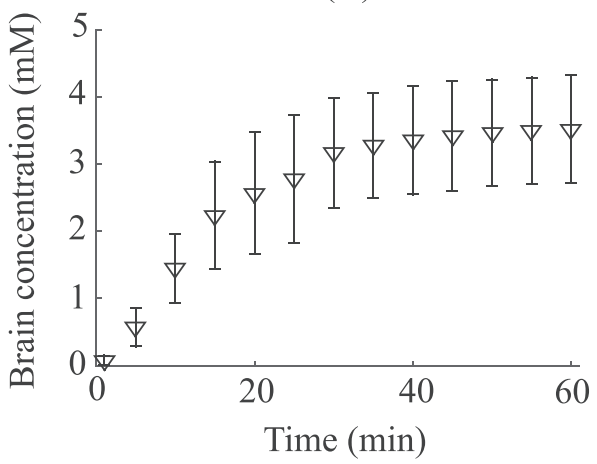

(B)

FIGURE 1 (a) optimized acetate infusion protocol in the rat used to obtain a stable acetate concentration in the brain after 30 min. (B) time course of acetate concentration in the brain [measured from in vivo ${ }^{1} \mathrm{H}$ nuclear magnetic resonance (NMR) spectra at $14.1 \mathrm{~T}$ ) following continuous intravenous acetate infusion in the rat. Each time point and its error bars present the average and standard deviation of seven animals, estimated by interpolation between the measured time points

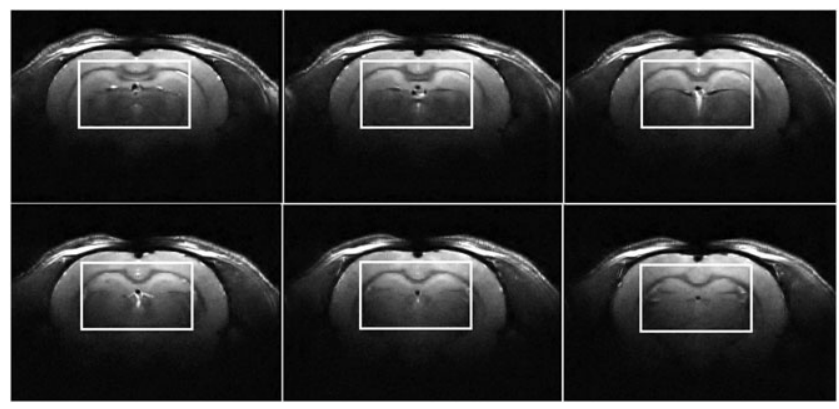

FIGURE 2 Diffusion-weighted magnetic resonance spectroscopy (DW-MRS) region of interest, with a size of $6 \times 10 \times 4 \mathrm{~mm}^{3}$, overlapped on axial images of the rat brain. Images acquired using fast spin echo multiple slice [field of view (FOV), $25 \times 25 \mathrm{~mm}^{2}$; slice thickness, $0.5 \mathrm{~mm}$; average, 4 ; data matrix, $128 \times 128$ ] at $14.1 \mathrm{~T}$

\section{3 | In vivo and in vitro NMR spectroscopy at $14.1 \mathrm{~T}$}

Spectra were acquired on a 14.1-T MRI system interfaced to a 26-cm horizontal bore magnet (Magnex Scientific, Oxford, UK; Varian, Palo Alto, CA, USA), equipped with an actively shielded gradient (inner diameter, $12 \mathrm{~cm}$ ) reaching $400 \mathrm{mT} / \mathrm{m}$ in $120 \mu \mathrm{s}$. A home-built quadrature transceiver with two physically decoupled 12-mm-diameter loops was used.

After initial setting, fast spin echo images [TR $=4 \mathrm{~s}$ and effective echo time $(T E)=40 \mathrm{~ms}$; four echoes] were acquired to select a volume of interest (VOI) of $450 \mu \mathrm{L}$ in the rat brain (Figure 2). Magnetic field homogeneity was adjusted using FAST(EST)MAP. ${ }^{27}$ Localized ${ }^{1} \mathrm{H}$ NMR spectra were acquired using the stimulated echo acquisition mode (STEAM) sequence. ${ }^{23}$ Acquisition parameters were optimized for acetate detection and are described further below.

Water suppression was achieved with seven consecutive chemical shift-selective (CHESS) adiabatic radiofrequency (RF) pulses followed by crusher gradients. Outer volume suppression was performed using eight adiabatic pulses followed by crusher gradients, which were repeated three times during the CHESS preparation to minimize the unwanted signal. One spectrum was acquired with the same VOI, but without application of water suppression, in order to provide a signal reference for quantification of the metabolites. 


\subsection{Accurate detection and quantification of acetate in ${ }^{1} \mathrm{H}$ NMR spectra}

To distinguish the overlapping acetate ${ }^{1} \mathrm{H}$ MRS resonance $(1.90 \mathrm{ppm})$ from the GABA resonance at 1.89 ppm, the rationale was to minimize the GABA signal by playing with $J$ modulation, enabling accurate acetate concentration quantification. As $J$ modulation is affected by both TE and mixing time (TM) in the STEAM sequence, a series of experiments was implemented to establish the optimum acquisition parameters. Experiments were performed in a phantom containing 50 mM GABA (Sigma-Aldrich, Shanghai, China) and 50mM NAA (Sigma-Aldrich, Milan, Italy) as a reference with $\mathrm{pH}$ adjusted to 7.2 and a temperature of $37.5^{\circ} \mathrm{C}$ in order to mimic in vivo conditions. Spectra were initially acquired with TE ranging from 2.8 to $100 \mathrm{~ms}$. With the optimum TE determined by the minimum amplitude of the GABA resonance at 1.89 ppm, a second series of data was acquired with TM ranging from 24 to $174 \mathrm{~ms}$. The optimum TM and TE values for the minimization of the GABA peak in phantom experiments were evaluated in in vivo experiments in the rat brain.

\section{5 | Diffusion weighting acquisition parameters}

The diffusion-weighted STEAM (DW-STEAM) sequence described by Pfeuffer et al. ${ }^{23}$ and Kunz et al. ${ }^{28}$ was used to investigate the metabolite diffusivity characteristics in vivo in the brain of seven adult rats. Diffusion gradients are placed during the time delay $\tau$ in the STEAM sequence: $90^{\circ}-\tau-90^{\circ}-\mathrm{TM}-90^{\circ}-\tau-\mathrm{ACQ}$. The signal attenuation of the ${ }^{1} \mathrm{H}$ diffusion is determined by the following acquisition parameters: diffusion gradient

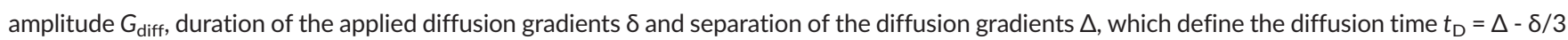
and give rise to $a b$-value of $b=\left(\gamma^{2} \cdot G^{2} \cdot \delta^{2}\right) \cdot t_{D}$. All of the gradient pulses, including both diffusion and STEAM gradients, were considered in the $b$-value calculation using a home-built Matlab script (The MathWorks, Natick, MA, USA). Diffusion gradients were applied in three orthogonal directions simultaneously in order to reach higher diffusion weighting. To vary the diffusion weighting of the signal in this study, only the diffusion gradient amplitude was changed, whereas the other parameters were kept constant.

To test the performance of the DW-STEAM sequence with the acquisition parameters optimized for the in vivo detection of acetate (as described in Section 3.1), the attenuation of the signal from a high-viscosity phantom containing 1,2-propanediol (Sigma-Aldrich, Buchs SG, Switzerland) at room temperature (around $20^{\circ} \mathrm{C}$ ) was measured by increasing the $b$-value up to $18 \mathrm{~ms} / \mu \mathrm{m}^{2}$.

In a first set of experiments, in vivo data were acquired with $G_{\text {diff }}=0,28,49,65$ and $91 \mathrm{mT} / \mathrm{m}$, corresponding to $b$-values ranging from 0.2 to $5 \mathrm{~ms} / \mu \mathrm{m}^{2}$. A second set of experiments to investigate the slow diffusion regime used three additional diffusion gradient amplitudes with stronger intensities of 137, 159 and $191 \mathrm{mT} / \mathrm{m}$ and corresponding $b$-values of 10,13 and $18 \mathrm{~ms} / \mathrm{\mu m}^{2}$. In all experiments, the signals were acquired with $\delta=5 \mathrm{~ms}, t_{\mathrm{D}}=73 \mathrm{~ms}, \mathrm{TR}=4 \mathrm{~s}$, and optimum TM and TE found to minimize the GABA resonance at 1.89 ppm in the STEAM sequence (as described in Section 3.1).

The DW-STEAM sequence was synchronized with the rat's breathing cycle with a respiration trigger in order to reduce the phase variation between the averaged free induction decays (FIDs) caused by movement, and the data of each scan were stored separately to allow the phase correction of individual spectra prior to averaging. To minimize the biasing effect of the attenuated spectral signals as a result of minor variations in physiology, acetate concentration, shimming or spectral quality during the experiment, the acquisition over the entire $b$-value range (0-18 ms/ $\mu \mathrm{m}^{2}$ ) for every animal was split into a series of acquisition sets with interleaved $b$-values. The number of replicate scans in every set was 16 FIDs for $0<b \leq 5 \mathrm{~ms} / \mu \mathrm{m}^{2}$ (each $1 \mathrm{~min} 5 \mathrm{~s}$ ), 32 FIDs for $b=10$ and $13 \mathrm{~ms} / \mu \mathrm{m}^{2}$ (each $2 \mathrm{~min} 10 \mathrm{~s}$ ) and 48 FIDs for $b=18 \mathrm{~ms} / \mu \mathrm{m}^{2}(3 \mathrm{~min} 15 \mathrm{~s}$ ).

\subsection{Processing and quantification of the data}

To prepare the acquired spectra from individual animals for analysis, the phase variations were corrected scan by scan by maximizing the NAA resonance height; then, the phased spectra acquired at the same $b$-value were corrected for $B_{0}$ drift and averaged together.

In vivo ${ }^{1} \mathrm{H}$ NMR summed spectra were processed using LCModel (Stephen Provencher Inc., Oakville, ON, Canada) and the unsuppressed water signal acquired from the same voxel was used as a reference for eddy current correction and determination of the apparent metabolite concentrations. No baseline correction, zero filling or apodization functions were applied to the data prior to the quantification. The Cramér-Rao lower bounds (CRLBs) calculated by LCModel were used as a measure of the reliability of the metabolite estimates.

The basis sets required by LCModel for spectral quantification were generated by simulations of all the metabolites detected in the spectra of the rat brain during continuous intravenous acetate infusion. The metabolite signals were simulated using the NMRSCOPE B plugin in JMRUI (http:// www.jmrui.eu) with the appropriate chemical shift and J-coupling patterns as described by Govindaraju et al. ${ }^{29,30}$ and STEAM sequence parameters used in this study. To validate the J-modulation characteristics of the simulated spectra, a series of in vitro experiments, using a phantom containing $50 \mathrm{mM}$ glutamine (Sigma Aldrich, Saint-Quentin-Fallavier, France) and $25 \mathrm{mM}$ creatine (Cr) (Sigma Aldrich, Buchs SG, Switzerland), was performed, varying TE from 2.8 to $100 \mathrm{~ms}$ with steps of $10 \mathrm{~ms}$ or less ( $\mathrm{TM}=20 \mathrm{~ms}$ and $\mathrm{TR}=10 \mathrm{~s}$ ). The macromolecule (MM) signals were measured experimentally in vivo in the rat brain with aTR of $4 \mathrm{~s}$ and the optimized values of TE and TM found for the accurate quantification of acetate (as described in Section 3.1). The measurement was performed by preceding the DW-STEAM sequence with an inversion recovery (IR) as described by Kunz et al. ${ }^{28}$ IR with a non-selective, full-passage, hyperbolic secant RF pulse and inversion time (TI) of 1 s provided the best suppression of the metabolite signal with the acquisition parameters used in this study (data not shown). To attenuate the residual metabolite signal after IR, diffusion gradients with a strength of $300 \mathrm{mT} / \mathrm{m}$ were applied along the three orthogonal directions simultaneously, resulting in a $b$-value of $41 \mathrm{~ms} / \mu \mathrm{m}^{2}$. 


\section{7 | Diffusion weighting attenuation modeling}

To allow comparison with previous in vivo diffusion studies of metabolites, ${ }^{22,23,31-33}$ apparent mono-exponential diffusion coefficients ( $D_{\text {mono }}^{\text {App }}$ ) of metabolites were estimated. Data for NAA and acetate in a $b$-value interval of 0-5 ms $/ \mathrm{mm}^{2}$ were fitted with a mono-exponential diffusion model using the following equation, where $S$ and $S_{0}$ are the signal intensities with and without diffusion gradients, respectively:

$$
\frac{S}{S_{0}}=\exp \left(-b \cdot D_{m o n o}^{A p p}\right)
$$

Diffusion-weighted data were then analyzed over $b$-values ranging from 0 to $18 \mathrm{~ms} / \mu \mathrm{m}^{2}$ and fitted using the following bi-exponential equation, where $D_{\text {fast }}^{\text {App }}$ and $D_{\text {slow }}^{\text {App }}$ represent the apparent diffusion coefficients (ADCs) of the fast and slow decaying signal components of each metabolite and $p_{\text {slow }}^{\text {App }}$ reflects the relative contribution of the slow component to the metabolite signal ${ }^{23}$ :

$$
\frac{S}{S_{0}}=p_{\text {slow }}^{\text {App }} \cdot \exp \left(-b \cdot D_{\text {slow }}^{\text {App }}\right)+\left(1-p_{\text {slow }}^{\text {App }}\right) \cdot \exp \left(-b \cdot D_{\text {fast }}^{\text {App }}\right)
$$

To determine the confidence intervals of the adjusted parameters, Monte Carlo simulations were performed with 500 artificial datasets generated based on the bootstrapping method. ${ }^{34}$ Curve fitting based on the Levenberg-Marquardt algorithm and further numerical procedures were performed in Matlab using in-house scripts. The extracellular fraction of acetate $p_{0}^{\text {extra }}$ (Ace) was estimated by the following equation ${ }^{23}$ :

$$
p_{0}^{\text {extra }}(\text { Ace })=1-p_{\text {slow }}^{\text {App }}(\text { Ace }) \cdot\left(k_{\text {intra }}+1\right)
$$

where $k_{\text {intra }}$ was determined from the contribution ratio of fast and slow components of a known intracellular metabolite, like NAA, simplified as:

$$
\begin{gathered}
k_{\text {intra }}=\frac{p_{\text {fast }}^{\text {App }}(\mathrm{NAA})}{p_{\text {slow }}^{\text {App }}(\mathrm{NAA})}, p_{\text {fast }}^{\text {App }}(\mathrm{NAA})=1-p_{\text {slow }}^{\text {App }}(\mathrm{NAA}) \\
k_{\text {intra }}=\frac{1}{p_{\text {slow }}^{\text {App }}(\mathrm{NAA})}-1
\end{gathered}
$$

\section{3 | RESULTS}

The main goal of the present study was to assess the acetate diffusion properties in vivo in brain tissue and to compare them with those of a reference intracellular metabolite, NAA. To this end, the acetate infusion protocol and DW-STEAM sequence parameters were optimized to ensure a reliable assessment of the diffusion behavior at steady-state acetate concentration in the rat brain.

\subsection{Apparent concentration of acetate in brain tissue}

To minimize spectral signal loss as a result of macroscopic motion, respiration cycle triggering was applied during acquisition and each FID signal was stored separately. The high quality and signal-to-noise ratio (SNR) of the acquired ${ }^{1} \mathrm{H}$ spectra enabled us to apply phase correction to individual spectra before signal averaging. The higher sensitivity provided using a surface coil at high magnetic field (14.1 T) with efficient $B_{0}$ shimming using the FAST(EST) MAP method yielded a linewidth of $20-25 \mathrm{~Hz},{ }^{27}$ with a typical NAA singlet resonance SNR of 14 averaging 16 scans acquired with a voxel size of $240 \mathrm{\mu L}$.

To reduce the signal overlap between the acetate singlet and the GABA resonance at $1.89 \mathrm{ppm}$, a long TE of $50 \mathrm{~ms}$ and TM of $49 \mathrm{~ms}$ in the STEAM sequence were used (Figure 3). This resulted in well-resolved acetate resonance (1.90 ppm) in spatially localized ${ }^{1} \mathrm{H}$ NMR spectra with and without diffusion weighting (Figure 4 and Supplementary material). The NAA singlet SNR of the spectra used in this study was higher than 20 for all $b$-values $\left(0-18 \mathrm{~ms} / \mu^{2}\right.$ ), which was sufficient for a precise quantification of the detected metabolites (CRLB $\left.\leq 20\right)$.

To ensure detectable acetate concentrations, an optimized infusion protocol was used to raise brain acetate concentrations to measurable levels, whilst maintaining the animal within physiological limits throughout the study. The acetate concentration time course in the rat brain reached steady state in less than $30 \mathrm{~min}$ (Figure 1). Brain acetate concentration during continuous intravenous acetate infusion reached $3.5 \mathrm{mM}$ $\left( \pm 20 \%\right.$ ) at steady state (Figure 1 ), estimated after correction for $T_{1}$ and $T_{2}$ relaxation of the signal. ${ }^{35}$

\section{2 | Diffusion-weighted ${ }^{1} \mathrm{H}$ MRS}

The signal attenuation of 1,2-propanediol was ascertained to be mono-exponential with $b$-values ranging from 0 to $18 \mathrm{~ms} / \mu \mathrm{m}^{2}$, and its self-diffusion coefficient $\left(D_{\text {free }}\right.$ ) at room temperature (around $20^{\circ} \mathrm{C}$ ) was about $0.32 \pm 0.07 \mu \mathrm{m}^{2} / \mathrm{ms}$, close to the value reported in a recent study, ${ }^{36}$ demonstrating the reliability of the procedure used in this study. 

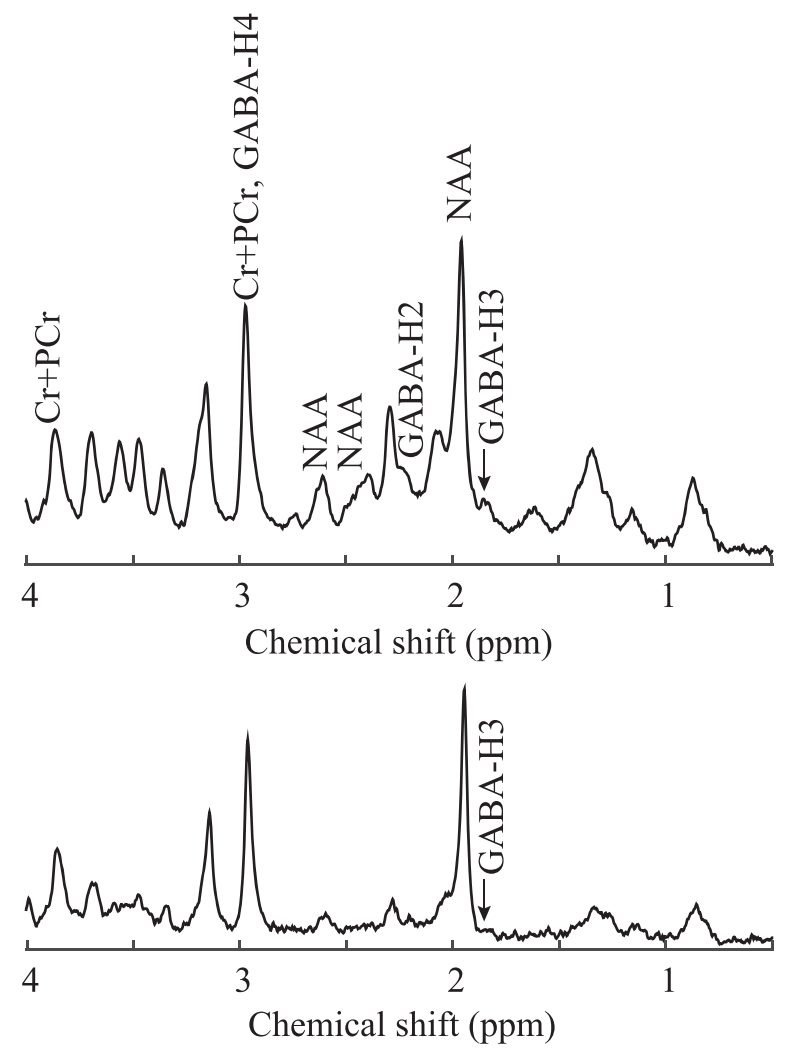

FIGURE 3 In vivo ${ }^{1} \mathrm{H}$ nuclear magnetic resonance (NMR) spectra of rat brain acquired using a stimulated echo acquisition mode (STEAM) sequence $(T R=4 \mathrm{~s}$ and apodization $=5 \mathrm{~Hz}$; average $=2 \times 16)$ in the absence of acetate infusion at short echo time $(\mathrm{TE}=8.5 \mathrm{~ms}$ and $\mathrm{TM}=20 \mathrm{~ms})($ top) and long echo time ( $\mathrm{TE}=50 \mathrm{~ms}$ and $\mathrm{TM}=49 \mathrm{~ms}$ ) (bottom). GABA-H4 and GABA-H2 indicate the triplet resonances at 3.01 and $2.28 \mathrm{ppm}$, and $\mathrm{GABA}$ $\mathrm{H} 3$ corresponds to the quintet resonance centered at $1.89 \mathrm{ppm}$. The noticeable shoulder corresponding to the quintet resonance of GABA at $1.89 \mathrm{ppm}$ was minimized at TE $50 \mathrm{~ms}$. $\mathrm{Cr}$, creatine; GABA, $\gamma$-aminobutyric acid; NAA, N-acetylphosphate; PCr, phosphocreatine

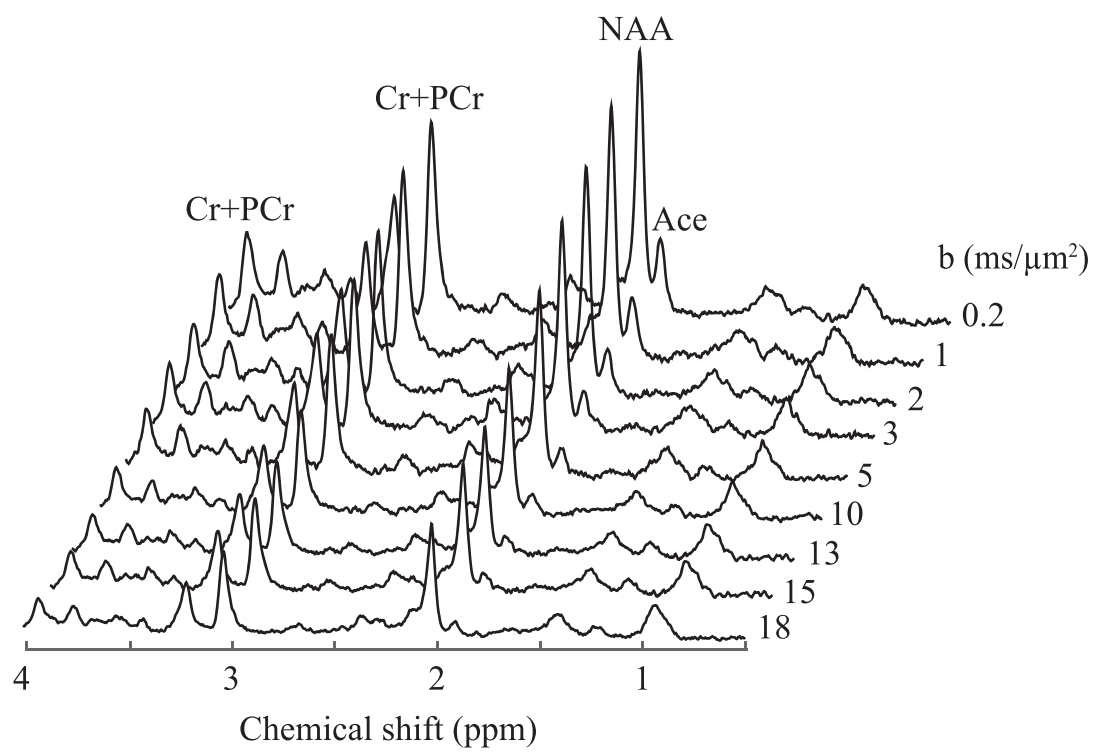

FIGURE 4 The diffusion-attenuated summed ${ }^{1} \mathrm{H}$ nuclear magnetic resonance (NMR) spectra (TR $=4 \mathrm{~s}, \mathrm{TE}=50 \mathrm{~ms}, \mathrm{TM}=49 \mathrm{~ms}$,

apodization $=5 \mathrm{~Hz}$ ) acquired during stability of acetate concentration in the single rat brain. Every spectrum is the sum of a given number of spectra (shown in parentheses) acquired at a specific b-value. From top to bottom: $0.2(4 \times 16), 1(4 \times 16), 2(4 \times 16), 3(4 \times 16), 5(4 \times 16), 10(4 \times 32), 13$ $(4 \times 32)$ and $18(4 \times 48) \mathrm{ms} / \mu \mathrm{m}^{2}$. Ace, acetate; $\mathrm{Cr}$, creatine; NAA, $\mathrm{N}$-acetylphosphate; $\mathrm{PCr}$, phosphocreatine

To compare the intrinsic diffusivity of acetate and NAA, the free diffusion of acetate and NAA was estimated in vitro in a phantom containing acetate and NAA with $b$-values ranging from 0 to $3.5 \mathrm{~ms} / \mu \mathrm{m}^{2}$. A mono-exponential fitting of the acquired DW signals resulted in a $D_{\text {free }}$ of $1.27 \pm 0.03 \mu \mathrm{m}^{2} / \mathrm{ms}$ for acetate and $0.83 \pm 0.02 \mu \mathrm{m}^{2} / \mathrm{ms}$ for NAA (Table 1 ). To determine the diffusivity of acetate and NAA for 2-3 h during 
continuous intravenous acetate infusion in rat, diffusion-attenuated spectra were acquired using the DW-STEAM sequence with optimized parameters (TE $=50 \mathrm{~ms}$ and $\mathrm{TM}=49 \mathrm{~ms}$ ). With increasing diffusion weighting up to $b=5 \mathrm{~ms} / \mu \mathrm{m}^{2}$ (Figure 4 and Supplementary material), the acetate intensity dropped by $65 \%$ and NAA by $35 \%$ of their respective intensities without diffusion weighting. Fitting a mono-exponential decay to the

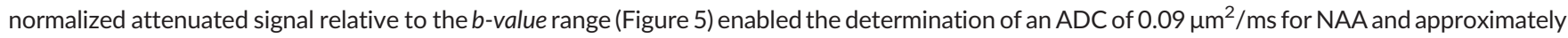
three times higher ADC for acetate, i.e. $0.24 \mu \mathrm{m}^{2} / \mathrm{ms}$, with a coefficient of variation lower than $5 \%$ (Table 1 ). When extending the measurement to higher $b$-values, the normalized diffusion-attenuated signal for NAA and acetate was clearly non-linear, indicating a multi-exponential decay (Figure 5). Increasing the diffusion weighting to higher $b$-values up to $18 \mathrm{~ms} / \mu \mathrm{m}^{2}$ resulted in signal attenuation of $83 \%$ for acetate and $63 \%$ for NAA compared with their initial amplitude. At maximum $b$-value, the NAA signal was still above the noise level of a single FID spectrum and the mean CRLBs estimated by LCModel for NAA and acetate quantification in summed spectra from a single animal were $2 \%$ and $10 \%$, respectively. In addition, other prominent brain metabolites, such as glutamate, taurine, $\mathrm{Cr}$ and phosphocreatine (PCr) (not addressed in this study), were well quantified with CRLBs of less than $10 \%$.

To characterize the non-mono-exponential decay of acetate and NAA, normalized attenuated metabolite signals were fitted using Equation 2 over the entire $b$-value range $\left(0-18 \mathrm{~ms} / \mu \mathrm{m}^{2}\right)$ in Figure 5 . Bi-exponential fitting provided ADCs for the fast and slow decaying components of the acetate and NAA signals (Table 2). The fast diffusion component of acetate showed an ADC ( $D_{\text {fast }}^{\text {App }}$ ) of $0.44 \pm 0.02 \mu \mathrm{m}^{2} / \mathrm{ms}$, whereas $D_{\text {fast }}^{\text {App }}$ of NAA was $0.20 \pm 0.04 \mu \mathrm{m}^{2} / \mathrm{ms}$. The slow diffusion component of acetate was characterized by an ADC ( $D_{\text {slow }}^{\text {App }}$ ) of $0.038 \pm 0.003 \mu \mathrm{m}^{2} / \mathrm{ms}$ and, for NAA, $D_{\text {slow }}^{\text {App }}$ was $0.026 \pm 0.010 \mu \mathrm{m}^{2} / \mathrm{ms}$. The probability distribution of the slow and fast diffusion of acetate and NAA (estimated from Monte Carlo simulations, $n=500$ ) is shown in Figure 6 .

The contribution of the slow component to the normalized attenuated signal ( $p_{\text {slow }}^{\text {App }}$ ) was found to be $33 \pm 2 \%$ for acetate and $58 \pm 10 \%$ for NAA. The intracellular component ratio $k_{\text {intra }}$ (Equation 5) was estimated from $p_{\text {slow }}^{\text {App }}$ of NAA $(0.72 \pm 0.1)$ and used as a reference for metabolites localized purely in the intracellular compartment. The extracellular fraction $p_{0}^{\text {extra }}$ for acetate (Equation 3 ) was estimated to be $43 \pm 12 \%$. Moreover, the estimation of $p_{0}^{\text {extra }}$ of acetate using $p_{\text {slow }}^{\text {App }}$ of $\mathrm{Cr}+\mathrm{PCr}$ instead of NAA yields a similar value of $39 \pm 8 \%$ (data not shown), encompassing the same range.

TABLE 1 Apparent diffusion coefficient of metabolites estimated using a mono-exponential fit in rat brain in vivo for $0<b<5 \mathrm{~ms} / \mu \mathrm{m}^{2}$ and in phantom for $0<b<3.5 \mathrm{~ms} / \mathrm{\mu m}^{2}$ (mean \pm standard deviation) calculated by Monte Carlo

\begin{tabular}{llr} 
& \multicolumn{2}{c}{$D_{\text {mono }}^{\text {App }\left(\mu \mathrm{m}^{2} / \mathrm{ms}\right)}$} \\
\cline { 2 - 3 } & \multicolumn{1}{c}{ Ace } & NAA \\
\hline In vivo & $0.24 \pm 0.008$ & $0.086 \pm 0.002$ \\
In phantom & $1.27 \pm 0.01$ & $0.83 \pm 0.01$ \\
\hline
\end{tabular}

Ace, acetate; NAA, N-acetylaspartate.

FIGURE 5 The normalized diffusionattenuated ${ }^{1} \mathrm{H}$ magnetic resonance spectroscopy (MRS) signal intensities of acetate (A) and $\mathrm{N}$-acetylaspartate (NAA) (B) in rat brain in vivo, with mono-exponential fitting of the $b$-value range of $0-5 \mathrm{~ms} / \mu \mathrm{m}^{2}$ (broken lines) and bi-exponential fitting of the $b$-value range of $0-18 \mathrm{~ms} / \mu \mathrm{m}^{2}$ (full lines) in the semilogarithmic plot. The error bars indicate the standard deviation of data averaged over seven animals

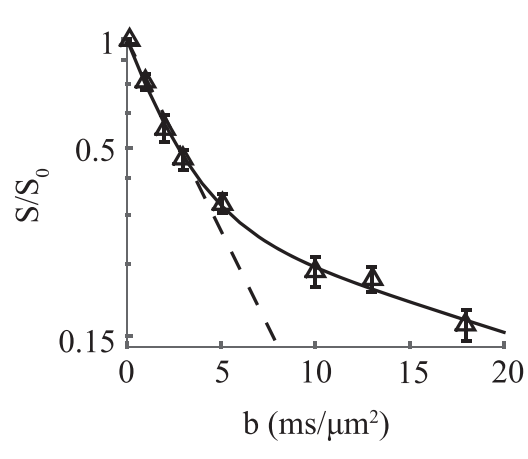

(A) Acetate

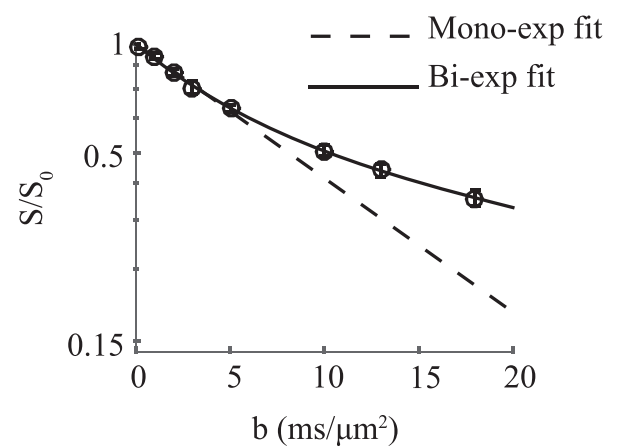

(B) NAA

TABLE 2 Apparent diffusion coefficients of metabolites in rat brain in vivo at $14.1 \mathrm{~T}$ estimated using a bi-exponential fit for $0<b<18 \mathrm{~ms} / \mu \mathrm{m}^{2}$ (mean \pm standard deviation) calculated by Monte Carlo

\begin{tabular}{lcr} 
& Ace & NAA \\
\hline$D_{\text {fast }}^{\text {app }}\left(\mu \mathrm{m}^{2} / \mathrm{ms}\right)$ & $0.44 \pm 0.02$ & $0.20 \pm 0.04$ \\
$D_{\text {slow }}^{\text {app }}\left(\mu \mathrm{m}^{2} / \mathrm{ms}\right)$ & $0.038 \pm 0.003$ & $0.026 \pm 0.010$ \\
$p_{\text {slow }}^{\text {app }}(\%)$ & $33 \pm 2$ & $58 \pm 10$
\end{tabular}

Ace, acetate; NAA, N-acetylaspartate. 


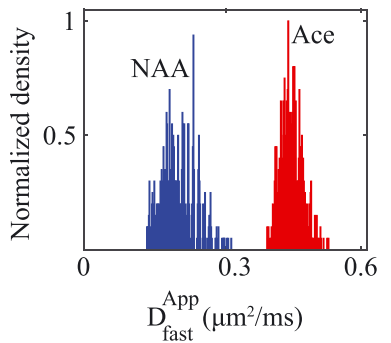

(A)

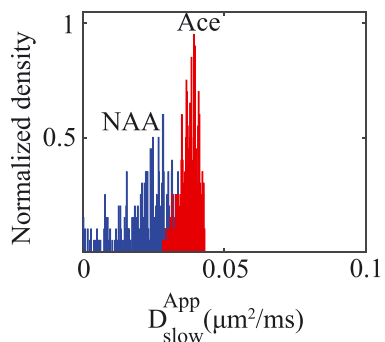

(B)

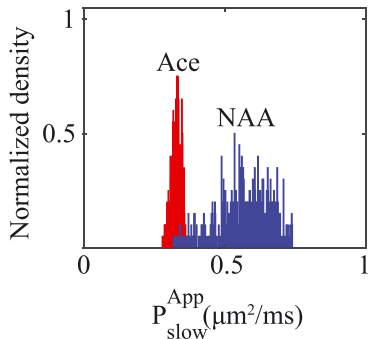

(C)

FIGURE 6 Monte Carlo probability distribution of the estimated parameters in the bi-exponential model including the apparent diffusivity of the fast component (a), apparent diffusivity of the slow component (B) and relative contribution of the slow component (C) in the total signal for acetate (ace) and $\mathrm{N}$-acetylaspartate (NAA). Artificial datasets $(n=500)$ in the Monte Carlo method were generated based on the bootstrapping method, i.e. every artificial data point corresponds to the average of seven points generated by sampling from measured dataset with replacement

\section{4 | DISCUSSION}

Localized ${ }^{1} \mathrm{H}$ NMR spectroscopy performed using a diffusion-weighted STEAM sequence at ultra-high magnetic field (14.1 T) allowed us to investigate the diffusivity characteristics of acetate and NAA in the rat brain in vivo. The diffusion behavior of acetate was compared with that of NAA, which is considered as a reference for the diffusivity characteristics of an exclusively intracellular metabolite. Observing the acetate signal at very high $b$-values implies the restriction of some part of the acetate signal to the intracellular space. The most notable result of the present work is the observation of significantly higher ADCs for acetate compared with NAA over the studied $b$-value range. The diffusion characteristics of acetate provide insight into the distribution volume of acetate in the rat brain.

\subsection{Precise quantification of acetate in the rat brain in vivo}

Acetate is present in brain tissue at concentrations too low to be consistently detectable by ${ }^{1} \mathrm{H}$ NMR in vivo, even at $14.1 \mathrm{~T}$, for the measurement of acetate transport across the blood-brain barrier and dynamic ${ }^{13} \mathrm{C}$ MRS of labeled acetate. ${ }^{1,8}$ The infusion protocol was optimized to provide a stable acetate concentration in vivo for extended time periods, which reached a readily detectable steady-state level of 3-4 mM (Figure 1).

The challenge to establish the reliable measurement of acetate diffusivity was to minimize the GABA resonance at 1.89 ppm which could interfere with the quantification of the acetate resonance, particularly at high diffusion weighting, as GABA is larger and less mobile than acetate. GABA has six NMR-observable protons in three methylene groups, forming an $\mathrm{A}_{2} \mathrm{M}_{2} \mathrm{X}_{2}$ spin system. The triplet resonances for GABA-H4 and GABA-H2 appear at 3.01 and 2.28 ppm, respectively, whereas the GABA-H3 quintet is centered at 1.89 ppm and thus overlaps with acetate. The acetate methyl resonance is a singlet peak and does not undergo $\mathrm{J}$-coupling evolution, whereas the GABA resonance could be suppressed using its unique J-coupling properties at a specific TE and TM. Therefore, application of the optimal parameters in the DW-STEAM sequence to minimize the GABA peak at $1.89 \mathrm{ppm}$ (TE $=50 \mathrm{~ms}$ and $\mathrm{TM}=49 \mathrm{~ms}$ ) allowed the quantification of acetate with minimal contamination from the GABA signal. However, the application of a long TE during ${ }^{1} \mathrm{H}$ MRS acquisition leads to substantial signal losses from $T_{2}$ relaxation and, accordingly, requires more averaging to achieve sufficient SNR for quantification.

With a TE of $50 \mathrm{~ms}$, CRLBs obtained from the LCModel quantification were lower than $10 \%$ for acetate and NAA in reference spectra, as well as in all diffusion-weighted spectra. The high precision of the estimated metabolite concentrations is ascribed to the high spectral resolution and SNR of the ${ }^{1} \mathrm{H}$ spectra acquired at $14.1 \mathrm{~T}$ and preprocessing to correct the phase and frequency drift of individual spectra before averaging and quantification of the metabolite signal.

The acquisition parameters (TE and TR) used in this study result in $T_{1}$ and $T_{2}$ relaxation effects on quantification of the measured signals, and this was taken into account and corrected using previously measured $T_{1}$ and $T_{2}$ relaxation times of the metabolites. ${ }^{35}$

\subsection{Diffusion characteristics of acetate and NAA}

To interpret the diffusivity of acetate without applying the assumptions used for diffusion data modeling (for reviews, see Cao \& Wu, ${ }^{13}$ Nicolay et al. ${ }^{14}$ and Ronen $\&$ Valette ${ }^{15}$ ), we compared it with the diffusion of NAA, as NAA is known to be an intracellular metabolite mostly confined to neurons, ${ }^{37}$ and therefore could be used for comparison with the diffusion properties of other metabolites, where the cellular localization may be less established. NAA is particularly suitable as a neuronal diffusion marker as it appears prominently in ${ }^{1} \mathrm{H}$ spectra of the rat brain, with its main singlet peak not subject to $J$ modulation.

When considering a range of $b$-values of $0-5 \mathrm{~ms} / \mu^{2}$, a mono-exponential decrease was observed, as judged from the linear decay for acetate and NAA in vivo signals in a semi-logarithmic plot (Figure 5). Furthermore, the application of respiration cycle triggering during ${ }^{1} \mathrm{H}$ spectral acquisition and single-FID phase correction generally avoided signal loss and the related overestimation of the metabolite ADC. ${ }^{38}$ The consistency of the estimated ADC of NAA using $b$-values in the range $0-5 \mathrm{~ms} / \mu^{2}{ }^{2}$ with previous studies performed at longer diffusion times ${ }^{23,33}$ implied that the 
diffusion time chosen in this study $(73 \mathrm{~ms}$ ) was long enough to place the diffusion signal decay of metabolites in the diffusion time independence regime. ${ }^{21}$ However, the ADC of NAA has been reported with slightly higher values in some studies, ${ }^{22,31}$ which could be explained by the shorter diffusion time used in these studies (10-20 ms). A shorter diffusion time implies a smaller mean displacement of metabolites, which limits the interaction with the environment and increases the ADC. ${ }^{18,39}$ In addition, the lower metabolite ADC values reported here could be ascribed to the higher magnetic field used (14.1 T) and the resulting higher sensitivity and spectral resolution.

The most noteworthy result of the present study is the observation of an approximately three-fold higher ADC of acetate compared with NAA for $b$-values in the range $0-5 \mathrm{~ms} / \mu \mathrm{m}^{2}$. This difference cannot solely be accounted for by the smaller molecular size and larger free diffusion of acetate, as its free ADC measured in a phantom was only 1.5 times that of NAA's, indicating that acetate is in an environment with fewer barriers to its diffusion.

The bi-exponential regression of normalized acetate and NAA signals over the $b$-value range $0-18 \mathrm{~ms} / \mu \mathrm{m}^{2}$ was characterized by fast and slow diffusion components $\left(D_{\text {fast }}^{\text {App }}, D_{\text {slow }}^{\text {App }}\right)$. The diffusion of the fast component of acetate was approximately two-fold higher than that of NAA, as observed in the mono-exponential fitting of the acetate and NAA diffusion signal in the $b$-value range $0-5 \mathrm{~ms} / \mu \mathrm{m}^{2}$ (Tables 1 and 2 ).

$D_{\text {slow }}^{\text {App }}$ was determined for acetate with higher precision than for NAA (error of $10 \%$ for acetate and $40 \%$ for NAA). In our study, the rationale was to choose a sufficiently large $b$-value range for the study of the hindered diffusion of acetate. Fortunately, the fast diffusion component of acetate decayed rapidly and its contribution at $b$-values higher than $5 \mathrm{~ms} / \mu \mathrm{m}^{2}$ was less than $11 \%$ and reached a negligible level of $0.03 \%$ at $18 \mathrm{~ms} / \mu \mathrm{m}^{2}$. Therefore, the acquisition of spectra with a $b$-value range of $0-18 \mathrm{~ms} / \mu \mathrm{m}^{2}$ was sufficient to determine both fast and slow diffusion components of acetate inside the brain for bi-exponential modeling. However, the $b$-value range of $0-18 \mathrm{~ms} / \mu \mathrm{m}^{2} \mathrm{might}$ not be sufficiently high for a precise estimation of the slow component of NAA, and could thus result in an overestimation of the slow component.

The diffusion of acetate in the extracellular compartment is reflected mainly in the fast diffusion component, and its diffusion in the intracellular compartment can probably be associated with the slow diffusion component. Considering $D_{\text {fast }}^{\text {App }}$ of acetate as $0.44 \mathrm{~ms} / \mu \mathrm{m}^{2}$, the contribution of the extracellular signal to the total signal measured at a $b$-value higher than $5 \mathrm{~ms} / \mu \mathrm{m}^{2}$ was less than $10 \%$. Therefore, the observed signal at $b$-values higher than $5 \mathrm{~ms} / \mu \mathrm{m}^{2}$ could be considered mainly as the slow component, reflecting the slow diffusing intracellular fraction of molecules. $D_{\text {slow }}^{\text {App }}$ of acetate and NAA was an order of magnitude lower than their $D_{\text {fast }}^{\text {App }}$, as reported for several metabolites in previous studies. ${ }^{18,23,33}$ The much smaller values of $D_{\text {slow }}^{\text {App }}$ than $D_{\text {fast }}^{\text {App }}$ demonstrate that intracellular barriers and restrictions dominate the diffusion of these metabolites at such high $b$-values and long diffusion times.

The percentage of the acetate signal at a $b$-value of $18 \mathrm{~ms} / \mu \mathrm{m}^{2}$ was $16 \%$ of the total signal, approximately two-fold less than that observed for NAA, namely $37 \%$. The faster decay of the fast component of acetate diffusion relative to NAA and the lower contribution of the slow diffusion component of acetate at high $b$-values suggest that a significant part of the acetate signal arises from the extracellular compartment.

\subsection{Evidence of acetate localization in glial cells}

Acetate has been proposed as a potential glial marker because of its almost exclusive uptake in astrocytes. ${ }^{1,7,40}$ The estimated $D_{\text {slow }}^{\text {App }}$ of acetate was slightly higher than that of NAA in Table 2. However, at the diffusion time used in this study (73 ms), $D_{\text {slow }}^{\text {App }}$ of metabolites is expected to reflect cellular dimensions rather than the respective molecule size. Slight differences in the estimated $D_{\text {slow }}^{\text {ppp }}$ for acetate and NAA may therefore suggest a different cellular localization, such as inositol in astrocytes, where the different $D_{\text {slow }}^{\text {App }}$ of acetate from a neuronal marker, NAA, could be attributed to the different structure and shape of glial cytosol compared with neuronal cells. However, the difference between $D_{\text {slow }}^{\text {App }}$ of NAA and acetate did not reach statistical significance and further investigations at substantially higher precision are indicated.

Interestingly, $D_{\text {slow }}^{\text {App }}$ for inositol $\left(0.037 \pm 0.003 \mu \mathrm{m}^{2} / \mathrm{ms}\right)$, reported by Pfeuffer et al., ${ }^{23}$ is close to $D_{\text {slow }}^{\text {App }}$ of acetate. This similarity suggests that acetate and inositol may share a similar intracellular distribution space, which is probably different from that of NAA. It has been proposed in several studies using different measurement techniques that acetate and inositol are both located mainly in glial cells. ${ }^{3,40-43}$

\subsection{Distribution volume of acetate in rat brain tissue}

The extracellular fraction of the acetate signal in the brain was estimated to be $43 \pm 12 \%$ under the experimental conditions of the present study, i.e. continuous intravenous acetate infusion in the rat. The contribution of the acetate signal from cerebrospinal fluid and blood was assumed to be negligible because of the rapid signal loss at low $b$-values (free diffusion of acetate, $1.27 \pm 0.01 \mu \mathrm{m}^{2} / \mathrm{ms}$ ). The estimated extracellular fraction of the acetate signal, $43 \%$, is greater than two-fold the reported value for the extracellular fraction of glucose (17\%). ${ }^{23}$ Therefore, the difference in extracellular fraction of acetate and glucose implies a distribution of acetate distinctly different from the uniform brain glucose distribution. In other words, the intracellular and extracellular signals have approximately equal contributions to the total detected acetate signal.

After passing the blood-brain barrier, acetate will enter the intracellular or extracellular space, and may be either uniformly or unequally distributed between these compartments. Based on the results of this study, $43 \%$ of the acetate signal is assigned to the extracellular space, a region occupying approximately $20 \%$ of the volume of the whole brain. ${ }^{44}$ Assuming identical acetate concentrations in the extracellular compartment and in the intracellular compartment in which acetate is localized, it follows that the intracellular fraction of acetate (57\%) is in a cellular compartment occupying approximately $27 \%$ of the brain, suggesting that acetate may not have access to the whole intracellular space, but rather to a subpopulation of 
cells in the brain. With this assumption, the acetate physical distribution volume $\left(V_{d}\right)$, i.e. the volume to which acetate has access, including the extracellular and/or intracellular space in the brain, is estimated to be $47 \%$.

Alternatively, with its metabolic conversion, acetate may have a lower steady-state concentration in the intracellular space compared with the extracellular space. In this case, acetate will be distributed in a larger intracellular volume than that estimated above assuming equal concentrations (27\%) and, consequently, acetate in both astrocytes and neurons would contribute to the intracellular signal. It is challenging to differentiate the concentration of acetate in different intracellular compartments because of the variable expression of monocarboxylate transporter (MCT) isoforms in astrocytes and neurons. ${ }^{44}$ Nonetheless, this simplified assumption, of an equal acetate concentration in neurons and astrocytes, would result in an extracellular acetate concentration three times the intracellular level.

Although our data cannot exclude this possibility, we believe that the results are best explained by acetate having access to an intracellular volume less than the whole intracellular space, which is consistent with acetate being a marker for glial metabolism as suggested in previous studies. ${ }^{1,7,40}$ The lower estimated value of $V_{d}$ for acetate in the rat brain is comparable with the acetate $V_{d}$ value (32 $\left.\pm 12 \%\right)$ reported by Patel et al. ${ }^{7}$ in a study using ${ }^{13} \mathrm{C}$-acetate. There, a smaller $V_{d}$ for acetate than for glucose was found to achieve better fitting of ${ }^{13} \mathrm{C}$-labeling time courses of glutamate and glutamine, as well as the time course of acetate concentration in brain in a kinetic analysis of acetate transport and utilization across the blood-brain barrier.

To acquire data in this study, the sequence parameters (e.g. TE and $b$-value) were optimized for precise quantification and evaluation of the diffusivity of acetate in the brain. Therefore, these parameters may not be optimal for the assessment of the diffusion behavior of other metabolites in the brain, such as NAA. $D_{\text {fast }}^{\text {App }}$ and $D_{\text {slow }}^{\text {App }}$ of NAA are determined with lower precision compared with acetate (cf. Figure 6) or with a previous study by Pfeuffer et al., ${ }^{23}$ where a much broader $b$-value range of $0-50 \mathrm{~ms} / \mu \mathrm{m}^{2}$ was used. The acquisition of spectra with sufficient SNR at a $b$-value of $50 \mathrm{~ms} / \mu^{2}$ requires more temporal averaging and results in a substantial increase in acquisition time.

The exchange of metabolites across the cell membrane could attenuate the intracellular diffusion signal, as has been reported for water diffusion inside the cell. ${ }^{45}$ The transport of acetate across the cell membrane was assumed to have a minor impact on the intracellular attenuated signal at the given diffusion time used in this study.

\section{5 | CONCLUSIONS}

We conclude that the presence of acetate resonance in ${ }^{1} \mathrm{H}$ spectra acquired at very large diffusion weighting indicates restricted diffusion of acetate in the intracellular region of brain. The different behavior of the slow diffusion component of acetate in the intracellular space compared with the neuronal marker NAA may suggest different intracellular localization for acetate in the brain, compared with NAA, most probably the glial compartment. It remains a challenge to link the bi-exponential model with the structure of compartments in the brain and the results should be considered with more caution.

Finally, we propose that acetate probably occupies a different physical distribution space than water and some other metabolites, such as glucose. The revised volume of distribution value of acetate may have a significant effect on the results and interpretations of acetate metabolism in the normal brain, as well as in clinical and preclinical studies of diseases such as diabetes.

\section{ACKNOWLEDGEMENTS}

This work was supported by the Swiss National Science Foundation (grant 149983) and by the Centre d'Imagerie BioMédicale (CIBM) of the École polytechnique fédérale de Lausanne (EPFL), Université de Lausanne (UNIL), Université de Genève (UNIGE), Hôpitaux Universitaires de Genève (HUG), and Centre hospitalier universitaire vaudois (CHUV).

\section{REFERENCES}

1. Lanz B, Xin L, Millet $\mathrm{P}$, Gruetter R. In vivo quantification of neuro-glial metabolism and glial glutamate concentration using ${ }^{1} \mathrm{H}-\left[{ }^{13} \mathrm{C}\right] \mathrm{MRS}$ at $14.1 \mathrm{~T}$. J Neurochem. 2014;128:125-139.

2. Cruz NF, Lasater A, Zielke HR, Dienel GA. Activation of astrocytes in brain of conscious rats during acoustic stimulation: Acetate utilization in working brain. J Neurochem. 2005;92:934-947.

3. Waniewski RA, Martin DL. Preferential utilization of acetate by astrocytes is attributable to transport. J Neurosci. 1998;18:5225-5233.

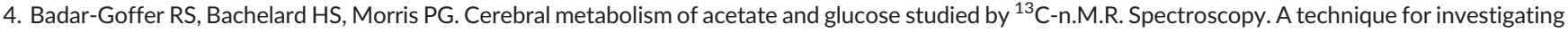
metabolic compartmentation in the brain. Biochem J. 1990;266:133-139.

5. Cerdan S, Künnecke B, Seelig J. Cerebral metabolism of $\left[1,2-{ }^{13} \mathrm{C}_{2}\right]$ acetate as detected by in vivo and in vitro ${ }^{13} \mathrm{C} \mathrm{NMR}$. J Biol Chem. 1990;265:12916-12926.

6. Nicklas WJ, Clarke DD. Decarboxylation studies of glutamate, glutamine, and aspartate from brain labelled with $\left[1-{ }^{14} \mathrm{C}\right]$ acetate, L-[U- $\left.{ }^{14} \mathrm{C}\right]-$ aspartate, and L-[U- ${ }^{14}$ C]glutamate. J Neurochem. 1969;16:549-558.

7. Patel AB, de Graaf RA, Rothman DL, Behar KL, Mason GF. Evaluation of cerebral acetate transport and metabolic rates in the rat brain in vivo using ${ }^{1} \mathrm{H}$ $\left[{ }^{13}\right.$ C]-NMR. J Cereb Blood Flow Metab. 2010;30:1200-1213.

8. Deelchand DK, Shestov AA, Koski DM, Uğurbil K, Henry P-G. Acetate transport and utilization in the rat brain. J Neurochem. 2009;109:46-54. 
9. Gruetter R, Adriany G, Choi IY, Henry PG, Lei H, Öz G. Localized in vivo ${ }^{13} \mathrm{C}$ NMR spectroscopy of the brain. NMR Biomed. 2003;16:313-338.

10. Henry P-G, Adriany G, Deelchand D, et al. In vivo ${ }^{13} \mathrm{C}$ NMR spectroscopy and metabolic modeling in the brain: A practical perspective. J Magn Reson Imaging. 2006;24:527-539.

11. Khan SA, Cox IJ, Hamilton G, Thomas HC, Taylor-Robinson SD. In vivo and in vitro nuclear magnetic resonance spectroscopy as a tool for investigating hepatobiliary disease: A review of ${ }^{1} \mathrm{H}$ and ${ }^{31} \mathrm{P}$ MRS applications. Liver Int. 2005;25:273-281.

12. Murphy E, Hellerstein M. Is in vivo nuclear magnetic resonance spectroscopy currently a quantitative method for whole-body carbohydrate metabolism? Nutr Rev. 2000;58:304-314.

13. Cao P, Wu EX. In vivo diffusion MRS investigation of non-water molecules in biological tissues. NMR Biomed. 2016;30:e3481.

14. Nicolay K, Braun KPJ, de Graaf RA, Dijkhuizen RM, Kruiskamp MJ. Diffusion NMR spectroscopy. NMR Biomed. 2001;14:94-111.

15. Ronen I, Valette J. Diffusion-weighted magnetic resonance spectroscopy. eMagRes. 2015;4:733-750.

16. Van Zijl PC, Moonen CT, Faustino P, Pekar J, Kaplan O, Cohen JS. Complete separation of intracellular and extracellular information in NMR spectra of perfused cells by diffusion-weighted spectroscopy. Proc Natl Acad Sci U S A. 1991;88:3228-3232.

17. Abe O, Okubo T, Hayashi N, et al. Temporal changes of the apparent diffusion coefficients of water and metabolites in rats with hemispheric infarction: Experimental study of transhemispheric diaschisis in the contralateral hemisphere at 7 tesla. J Cereb Blood Flow Metab. 2000;20:726-735.

18. Assaf $\mathrm{Y}$, Cohen $\mathrm{Y}$. In vivo and in vitro bi-exponential diffusion of $\mathrm{N}$-acetyl aspartate (NAA) in rat brain: A potential structural probe? NMR Biomed. 1998;11:67-74.

19. de Graaf RA, Braun KP, Nicolay K. Single-shot diffusion trace (1)H NMR spectroscopy. Magn Reson Med. 2001;45:741-748.

20. Marchadour C, Brouillet E, Hantraye P, Lebon V, Valette J. Anomalous diffusion of brain metabolites evidenced by diffusion-weighted magnetic resonance spectroscopy in vivo. J Cereb Blood Flow Metab. 2012;32:2153-2160.

21. Najac C, Marchadour C, Guillermier M, et al. Intracellular metabolites in the primate brain are primarily localized in long fibers rather than in cell bodies, as shown by diffusion-weighted magnetic resonance spectroscopy. Neuroimage. 2014;90:374-380.

22. Van der Toorn A, Dijkhuizen RM, Tulleken CAF, Nicolay K. Diffusion of metabolites in normal and ischemic rat brain measured by localized ${ }^{1} \mathrm{H}$ MRS. Magn Reson Med. 1996;36:914-922.

23. Pfeuffer J, Tkác I, Gruetter R. Extracellular-intracellular distribution of glucose and lactate in the rat brain assessed noninvasively by diffusion-weighted ${ }^{1} \mathrm{H}$ nuclear magnetic resonance spectroscopy in vivo. J Cereb Blood Flow Metab. 2000;20:736-746.

24. Burnier P, Tappy L, Jéquier E, Schneeberger D, Chioléro R. Metabolic and respiratory effects of infused sodium acetate in healthy human subjects. Am J Physiol. 1992;263:R1271-R1276.

25. Ward RA, Wathen RL, Harding GB, Thompson LC. Comparative metabolic effects of acetate and dichloroacetate infusion in the anesthetized dog. Metabolism. 1985;34:680-687.

26. Sugimoto H, Koehler RC, Wilson DA, Brusilow SW, Traystman RJ. Methionine sulfoximine, a glutamine synthetase inhibitor, attenuates increased extracellular potassium activity during acute hyperammonemia. J Cereb Blood Flow Metab. 1997;17:44-49.

27. Gruetter R, Tkác I. Field mapping without reference scan using asymmetric echo-planar techniques. Magn Reson Med. 2000;43:319-323.

28. Kunz N, Cudalbu C, Mlynarik V, Hüppi PS, Sizonenko SV, Gruetter R. Diffusion-weighted spectroscopy: A novel approach to determine macromolecule resonances in short-echo time ${ }^{1} \mathrm{H}$-MRS. Magn Reson Med. 2010;64:939-946.

29. Govindaraju V, Young K, Maudsley AA. Corrigendum: Proton NMR chemical shifts and coupling constants for brain metabolites. NMR Biomed. 2015;28:923-924.

30. Govindaraju V, Young K, Maudsley AA. Proton NMR chemical shifts and coupling constants for brain metabolites. NMR Biomed. 2000;13:129-153.

31. Duong TQ, Sehy JV, Yablonskiy DA, Snider BJ, Ackerman JJ, Neil JJ. Extracellular apparent diffusion in rat brain. Magn Reson Med. 2001;45:801-810.

32. Kunz N. Biophysical basis of the diffusion weighted magnetic resonance signal in the rat brain. Ph.D. thesis, École Polytechnique Fédérale de Lausanne, 2010.

33. Ligneul C, Palombo M, Valette J. Metabolite diffusion up to very high b in the mouse brain in vivo: revisiting the potential correlation between relaxation and diffusion properties. Magn Reson Med. 2016;77:1390-1398.

34. Press WH, Teukolsky SA, Vetterling WT, Flanner BP. Numerical Recipes: The Art of Scientific Computing. 3rd ed. New York: Cambridge University Press; 2007:691-692.

35. Dehghani MM, Kunz N, Gruetter R. T1 and T2 relaxation times of infused acetate in rat brain at 14.1 Tesla. ESMRMB. 32nd Annual Scientific Meeting. MAGMA. 2015;28:397-398.

36. D'Agostino C, Ryabenkova Y, Miedziak PJ, et al. Deactivation studies of a carbon supported AuPt nanoparticulate catalyst in the liquid-phase aerobic oxidation of 1,2-propanediol. Cat Sci Technol. 2014;4:1313-1322.

37. Moffett JR, Namboodiri MA, Cangro CB, Neale JH. Immunohistochemical localization of N-acetylaspartate in rat brain. Neuroreport. 1991;2:131-134.

38. Posse S, Cuenod CA, Le Bihan D. Human brain: Proton diffusion MR spectroscopy. Radiology. 1993;188:719-725.

39. Helmer KG, Dardzinski BJ, Sotak CH. The application of porous-media theory to the investigation of time-dependent diffusion in in vivo systems. NMR Biomed. 1995;8:297-306.

40. Wyss MT, Magistretti PJ, Buck A, Weber B. Labeled acetate as a marker of astrocytic metabolism. J Cereb Blood Flow Metab. 2011;31:1668-1674.

41. Brand A, Richter-Landsberg C, Leibfritz D. Multinuclear NMR studies on the energy metabolism of glial and neuronal cells. Dev Neurosci. 1993;15:289-298.

42. Minchin MCW, Beart PM. Compartmentation of amino acid metabolism in the rat dorsal root ganglion; a metabolic and autoradiographic study. Brain Res. 1975;83:437-449.

43. Muir D, Berl S, Clarke DD. Acetate and fluoroacetate as possible markers for glial metabolism in vivo. Brain Res. 1986;380:336-340. 
44. Nicholson C, Kamali-Zare P, Tao L. Brain extracellular space as a diffusion barrier. Comput Vis Sci. 2011;14:309-325.

45. Waniewski RA, Martin DL. Astrocytes and synaptosomes transport and metabolize lactate and acetate differently. Neurochem Res. 2004;29:209-217.

\section{SUPPORTING INFORMATION}

Additional Supporting Information may be found online in the supporting information tab for this article.

How to cite this article: Dehghani M, Kunz N, Lanz B, Yoshihara HAI, Gruetter R. Diffusion-weighted MRS of acetate in the rat brain. NMR in Biomedicine. 2017;30:e3768. https://doi.org/10.1002/nbm.3768 